\title{
Absorption coefficient for the intraband transitions in quantum dot materials
}

\author{
Antonio Luque, Antonio Martí, Alex Mellor , D. Fuertes Marrón, I. Tobías and E. Antolín
}

\begin{abstract}
In this paper, we present calculations of the absorption coefficient for transitions between the bound states of quantum dots grown within a semiconductor and the extended states of the conduction band. For completeness, transitions among bound states are also presented. In the separation of variables, single band $\mathrm{k} \cdot \mathrm{p}$ model is used in which most elements may be expressed analytically. The analytical formulae are collected in the appendix of this paper. It is concluded that the transitions are strong enough to provide a quick path to the conduction band for electrons pumped from the valence to the intermediate band. Copyright (C) 2012 John Wiley \& Sons, Ltd.
\end{abstract}

KEYWORDS

intermediate band solar cell; absorption; quantum dot; quantum calculations

\section{INTRODUCTION AND BACKGROUND}

Understanding intraband optical transitions in quantum dots (QDs) is of interest, for example, in semiconductor devices, such as the quantum dot intermediate band (IB) solar cell [1-3] or the quantum dot infrared photodetector [4,5] (QDIP). Models are very useful, not only for the simulation of the operation of these devices but also for providing an intellectual framework to help in their development. In the case of intraband transitions, that is, of transitions between bound states (BS) associated to the presence of the QD and between bound and extended states (ES) in the conduction band (CB), a good approximation can be obtained using the one-band $\mathrm{k} \cdot \mathrm{p}$ method [6]. This single band approximation is commonly used for phenomena involving only the CB. Actually, in the example studied in this paper, the projection of four-band $\mathrm{k} \cdot \mathrm{p} \mathrm{CB}$ fundamental state wavefunction onto the $\mathrm{CB}$ is $92 \%$, the remaining $8 \%$ being projected onto the virtual bound (VB) [7]. This means that the contribution of the VB components can be neglected with an error of about $8 \%$.

A model for intraband optical transitions between BSs has been presented elsewhere [8]. In this paper, we complete the model by including transitions between BSs and ESs in the CB.
In the one-band $\mathrm{k} \cdot \mathrm{p}$ method, the stationary wavefunctions are expressed in the form $\Psi=u_{0} \psi$, where $u_{0}$ is the CB Bloch function that solves the time-independent Schrödinger equation for the case of $k=0$ in the semiconductor and $\psi$ is the envelope function that solves the envelope equation

$$
-\frac{\hbar^{2}}{2 m_{\mathrm{c}}} \nabla^{2} \psi+U \psi=E \psi
$$

where $m_{\mathrm{c}}$ is the effective mass of electrons in the CB (which in zincblende lattices, such as GaAs and InAs, is approximately isotropic) and $U(r)$ represents the CB bottom, which is a variable with the composition of the semiconductor. For the calculations in this paper, we shall consider $U(r)$ to have a square well profile with zero potential inside the QD (the energy origin) and $U_{0}$ outside it.

The function $u_{0}$ has a fast spatial variation imposed by the periodicity of the crystal lattice, whereas the envelope function $\psi$ has a much slower variation comparable with the characteristic length of the nanostructures.

The most common way of producing QD devices is by molecular beam epitaxy in the Stransky Krastanov growth mode [9]. This method produces squat truncated quadrangular pyramids. For numerical ease, we treat the QD shape as a parallelepiped with a square base of widths $2 a=2 b$ and height $2 c$, the latter being usually smaller. This is in agreement with some authors [10,11] and follows from 
our previous work [8].A transmission electron microscopy micrograph of a Stransky Krastanov grown QD with the modeled shape superimposed is shown in Figure 1. We believe that the strict shape is not critical to the accuracy of the model. In fact, other authors consider the QDs to be lens-shaped [12,13]. Concerning the envelope functions associated to the QDs, we consider that the function can be written as $\psi(\boldsymbol{r})=X(x) Y(y) Z(z)$, where each factor is the solution of a one-dimensional equation of the form of Eq. (1) and in which the potential function is a square potential well of height $U_{0}$ and width $2 a$, $2 b$ or $2 c$. Details on how this solution approaches the exact solution are given elsewhere [8]. The factorized solution obtained by separation of variables is very accurate for squat QDs but less so when $a=b \cong c$. The use of the separation of variables method simplifies the calculations significantly.

\section{EIGENVALUES AND EIGENFUNCTIONS}

\subsection{One-dimensional solutions}

In the following, we only consider the function $X(x)$, though $Y(y)$ and $Z(z)$ are analogous. Because of the symmetry of the potential $U_{0}$, all solutions $X(x)$ can be classified as odd or even. Solutions with energies less than $U_{0}$ are of the form $\cos (k x)$ (even solutions) or $\sin (k x)$ (odd solutions) within the well flanked by two fading exponential functions outside it. Taking the bottom of the well to be the energy origin, the dispersion relation is

$$
k^{2}=\left(2 m_{\mathrm{c}} / \hbar^{2}\right) E
$$

Permitted values of $k$ are those which assure no unbounded exponential exists (see reference [8]). The fading coefficient is $\kappa^{2}=\left(2 m_{\mathrm{c}} / \hbar^{2}\right)\left(U_{0}-E\right)$. These states are called BS.

For energies above the well $\operatorname{rim}\left(E>U_{0}\right)$, the solutions have the same expression within the well but are flanked by harmonic functions with $k_{\mathrm{e}}^{2}=\left(2 m_{\mathrm{c}} / \hbar^{2}\right)\left(E-U_{0}\right)$. In this case, the all-harmonic solution is an ES, and there is no restriction on $k$; the energy spectrum is continuous. Strictly speaking, the ESs of the continuous spectrum cannot be normalized because the normalization constant

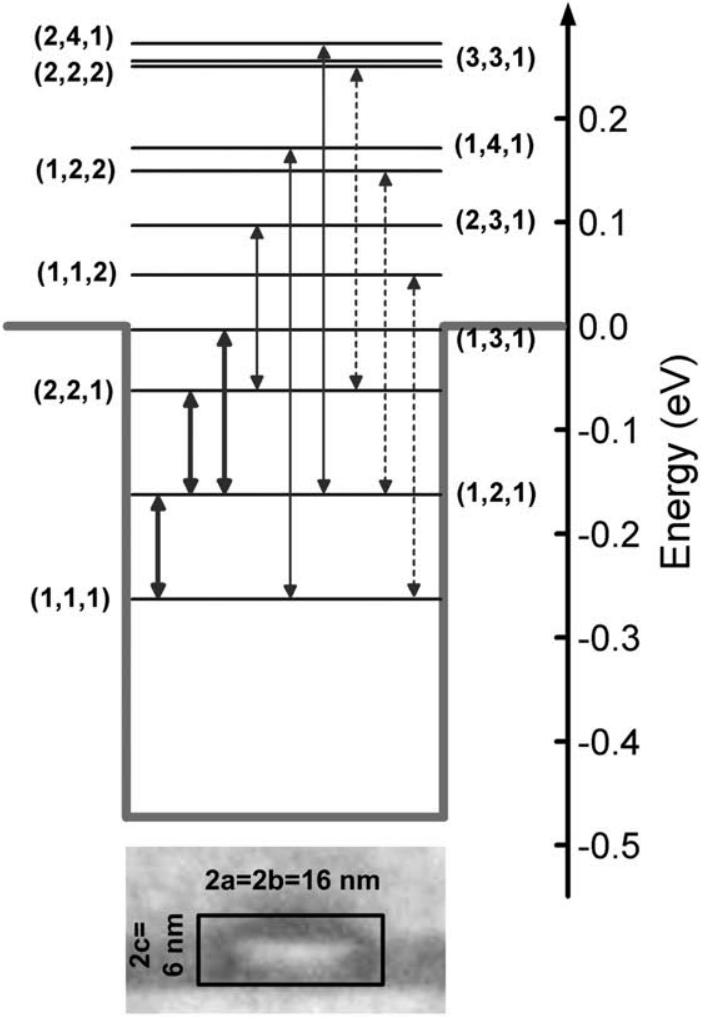

Figure 1. Above: energy levels of the bound states for the quantum dot parameters defined in Table I. Below: transmission electron microscopy micrograph of one of the quantum dots with the modeled shape superimposed. This figure has been reproduced from [7] with permission.

flanked outside the potential well by solutions of the type $\cosh (\kappa(L-x))$ and $\sinh (\kappa(L-x))$ for even and odd functions, respectively. The conditions of continuity of the function and its first derivative at the well boundaries lead to the equations

$$
\begin{gathered}
\cot (k a)=(k / \kappa) \operatorname{coth}(\kappa(L-a)) \\
-\tan (k a)=(k / \kappa) \tanh (\kappa(L-a))
\end{gathered}
$$

for even and odd solutions, respectively. For very large $L(\kappa(L-a)>7)$, this condition is the same as in [8] for an unlimited space. In our calculations, $L$ is chosen as to ensure this condition is fulfilled. For harmonic unbounded solutions, with energies greater than $U_{0}$, the condition at the well boundaries is

$$
\begin{aligned}
& \left(k_{\mathrm{e}} / k\right) \cot (k a)=\cot \left(k_{\mathrm{e}}(a-\theta)\right) \Rightarrow k_{\mathrm{e}}(a-\theta)=\arg \cot \left[\left(k_{\mathrm{e}} / k\right) \cot (k a)\right] \\
& \left(k_{\mathrm{e}} / k\right) \tan (k a)=\tan \left(k_{\mathrm{e}}(a-\theta)\right) \Rightarrow k_{\mathrm{e}}(a-\theta)=\arg \tan \left[\left(k_{\mathrm{e}} / k\right) \tan (k a)\right]
\end{aligned}
$$

would be zero.

To avoid difficulties with the continuum, we shall restrict the one-dimensional space to a large but limited segment of length $2 L$ and apply periodic boundary conditions at the ends. Under this assumption, the BSs are again for even and odd solutions, respectively. For a given value of the energy, the parameter $\theta$ is adjusted to assure continuity at the well boundary.

The periodicity restricts the energy to discrete values. This implies 
$\cot \left(-k_{\mathrm{e}}(L-\theta)\right)=\cot \left(k_{\mathrm{e}}(L-\theta)\right)=0 \Rightarrow k(L-\theta)=(2 i+1) \pi / 2$

$\tan \left(-k_{\mathrm{e}}(L-\theta)\right)=\tan \left(k_{\mathrm{e}}(L-\theta)\right)=0 \Rightarrow k(L-\theta)=2 i \pi / 2$

where $i$ is an integer for even and odd solutions, respectively. In all cases, $k=j \pi / 2 L$ leads to an even function when $j$ is odd and to an odd function when $j$ is even. There is one allowed state per interval $\Delta k=\pi / 2 L$. In the same way, we can state that there is one odd and one even state in each $k$-interval of length

$$
\Delta k_{\mathrm{odd}}=\Delta k_{\mathrm{even}}=\pi / L
$$

For $\kappa(L-a)>7$, the norm of the bound functions is the same as for an unlimited space, which has been calculated already [8] (see Eq. (A1) in the Appendix). For extended functions, $N^{2}=L$ for very large values of $L$ (as compared with $d, 1 / k$ and $\theta$ ) independently of $j$ (provided $j \pi<<L$ ). In this case, the normalization constant for these functions is $L_{,}^{-1 / 2}$ which may be small but not strictly zero (see Eq. (A2) in the Appendix).

\subsection{Three-dimensional solutions}

The same calculation can be repeated for the other coordinates. We shall use subindices $x, y$, and $z$ to label the values of $k, k_{\mathrm{e}}, \kappa$ and $E$ for each coordinate. The total energy (considering the origin to be at the QD CB bottom) will be

$$
\begin{aligned}
E & =E_{x}+E_{y}+E_{z}=\hbar^{2}\left(k_{x}^{2}+k_{y}^{2}+k_{z}^{2}\right) / 2 m_{\mathrm{c}} \\
& =\hbar^{2} k^{2} / 2 m_{\mathrm{c}}
\end{aligned}
$$

leading to functions that can be bound in some coordinates and extended in others or totally bound or extended in all three coordinates. It is worth mentioning that, even if they are bound in the three coordinates, it may occur that their total energy be higher than $U_{0}$, These are known as virtual bound states $[14,15]$.

\section{ABSORPTION COEFFICIENT}

\subsection{Absorption coefficient and matrix elements}

In device physics, the carrier generation rate per unit volume and time is given by the expression [7]

$$
g=\int \alpha \mathrm{d} N_{\mathrm{ph}}=\int \frac{\mathrm{d} N_{\mathrm{ph}}}{\mathrm{d} E} \alpha \mathrm{d} E
$$

where $\mathrm{d} N_{\mathrm{ph}}$ is an elementary flux of photons per unit area and time in a narrow range of energies and $\alpha$ is the absorption coefficient. Following the discussion in [7], for transitions between states with wavefunctions $\psi$ and $\psi^{\prime}$, the exciton generation rate per unit volume and time is

$$
g=\int \frac{\mathrm{d} N_{\mathrm{ph}}}{\mathrm{d} E} \frac{2 \pi^{2} e^{2} E}{n_{\mathrm{ref}} c h \varepsilon_{0}} \frac{\left|\left\langle\psi|\mathbf{r} \cdot \boldsymbol{\varepsilon}| \psi^{\prime}\right\rangle\right|^{2}}{4 a b} F N_{l} \delta\left(E_{\text {line }}-E\right) \mathrm{d} E
$$

where $\boldsymbol{\varepsilon}$ is the light polarization vector, $n_{\text {ref }}$ is the refractive index of the medium, $F$ is the fractional coverage of the surface with QDs, $N_{l}$ is the number of QD layers per unit length, and $E_{\text {line }}$ is the energy difference between the two states. By analogy,

$$
\begin{aligned}
\alpha & =\frac{2 \pi^{2} e^{2} E}{n_{\mathrm{ref}} \operatorname{ch} \varepsilon_{0}} \frac{\left|\left\langle\psi|\mathbf{r} \cdot \boldsymbol{\varepsilon}| \psi^{\prime}\right\rangle\right|^{2}}{4 a b} F N_{l} \delta\left(E_{\text {line }}-E\right) \\
& \equiv \alpha^{\prime} E \delta\left(E_{\text {line }}-E\right)
\end{aligned}
$$

where $\alpha^{\prime}$ is defined for convenience. These values have to be doubled to account for the spin degeneracy of each state.

\subsection{Influence of polarization}

The matrix element $\left\langle\psi|\boldsymbol{\varepsilon} \cdot \mathbf{r}| \psi^{\prime}\right\rangle=\cos \varphi \sin \theta\left\langle\psi|x| \psi^{\prime}\right\rangle+$ $\sin \phi \sin \theta\left\langle\psi|y| \psi^{\prime}\right\rangle+\cos \theta\left\langle\psi|z| \psi^{\prime}\right\rangle$ depends on the photon polarization, which is defined here by its Euler angles $(\phi, \theta)$. Following the separation of variables, the matrix element $\left\langle\psi|x| \psi^{\prime}\right\rangle$ is the product three functions: one depending on $x$, one depending on $y$, and one depending on $z$. To have $\left\langle\psi|x| \psi^{\prime}\right\rangle \neq \mathbf{0}$, the quantum numbers $n_{y}$ and $n_{z}$ must be the same for $\psi$ and $\psi^{\prime}$ because otherwise their internal product is zero; therefore, only $n_{x}$ can vary. What is more, the quantum numbers must be of different parity, as required by selection rules in the electric dipole approximation. The same can be said for the other coordinates: the only nonzero matrix elements are those for which one single quantum number changes between two states of different parity.

The square of the module of the matrix element, entering in the absorption coefficient, can be written as follows:

$$
\begin{aligned}
\left|\left\langle\psi|\boldsymbol{\varepsilon} \cdot \mathbf{r}| \psi^{\prime}\right\rangle\right|^{2}= & \cos ^{2} \phi \sin ^{2} \theta\left|\left\langle\psi|x| \psi^{\prime}\right\rangle\right|^{2} \\
& +\sin ^{2} \phi \sin \theta^{2}\left|\left\langle\psi|y| \psi^{\prime}\right\rangle\right|^{2} \\
& +\cos \theta^{2}\left|\left\langle\psi|z| \psi^{\prime}\right\rangle\right|^{2} \\
& +2 \cos \phi \sin \phi \sin ^{2} \theta \operatorname{Re}\left[\left\langle\psi|x| \psi^{\prime}\right\rangle\left\langle\psi|y| \psi^{\prime}\right\rangle\right] \\
& +2 \cos \phi \sin \theta \cos \theta \operatorname{Re}\left[\left\langle\psi|x| \psi^{\prime}\right\rangle\left\langle\psi|z| \psi^{\prime}\right\rangle\right] \\
& +2 \sin \phi \sin \theta \cos \theta \operatorname{Re}\left[\left\langle\psi|y| \psi^{\prime}\right\rangle\left\langle\psi|z| \psi^{\prime}\right\rangle\right]^{2}
\end{aligned}
$$

Because only one quantum number may vary, the terms containing two different matrix elements are always zero. For instance, if the changing quantum number is $n_{x}$, then $\left\langle\psi|y| \psi^{\prime}\right\rangle$ is zero and so is the product $\left\langle\psi|x| \psi^{\prime}\right\rangle\left\langle\psi|y| \psi^{\prime}\right\rangle$. In consequence, 


$$
\begin{aligned}
\left|\left\langle\varphi|\boldsymbol{\varepsilon} \cdot \mathbf{r}| \varphi^{\prime}\right\rangle\right|^{2}= & \cos ^{2} \phi \sin ^{2} \theta\left|\left\langle\psi|x| \psi^{\prime}\right\rangle\right|^{2} \\
& +\sin ^{2} \phi \sin \theta^{2}\left|\left\langle\psi|\psi| y \mid \psi^{\prime}\right\rangle\right|^{2} \\
& +\cos \theta^{2}\left\langle\psi|z| \psi^{\prime}\right\rangle
\end{aligned}
$$

and only one of these three terms can be nonzero. and the first excited state associated to QDs are bound in all three coordinates. Transitions to other BSs are Dirac-delta shaped, following Eq. (10). Given that, for $\mathrm{a}=\mathrm{b}>\mathrm{c}$, the first excited states are the degenerate $(2,1,1)$ and $(1,2,1)$ states; the absorption coefficient for transitions from the fundamental or first excited state to all other BSs is

$$
\begin{aligned}
\alpha^{B S}= & 2 f_{1,1,1} \sum_{(n x, n y, n z) \neq(1,1,1)}\left(1-f_{n x, n y, n z}\right) E_{\text {line }} \alpha_{1,1,1}^{n x, n y, n z} \delta\left(E_{\text {line }}-E\right) \\
& +2 f_{1,2,1} \sum_{(n x, n y, n z) \neq(1,1,1),(1,2,1),(2,1,1)}\left(1-f_{n x, n y, n z}\right) E_{\text {line }} \alpha_{1,2,1}^{n n x, n y} \delta\left(E_{\text {line }}-E\right) \\
& +2 f_{2,1,1} \sum_{(n x, n y, n z) \neq(1,1,1),(1,2,1),(2,1,1)}\left(1-f_{n x, n y, n z}\right) E_{\text {line }} \alpha_{2,1,1}^{n n x, n y, n z} \delta\left(E_{\text {line }}-E\right)
\end{aligned}
$$

The incident photons have a range of polarizations, and the observed data results from averaging $\left|\left\langle\psi|\mathbf{r} \cdot \boldsymbol{\varepsilon}| \psi^{\prime}\right\rangle\right|^{2}$ . In the case of normal illumination, because the electric field of the photons is transversal, the polarization vector has $\theta=\pi / 2$ and $\left|\left\langle\psi|\mathbf{r} \cdot \boldsymbol{\varepsilon}| \psi^{\prime}\right\rangle\right|^{2}=\cos ^{2} \phi\left|\left\langle\psi|x| \psi^{\prime}\right\rangle\right|^{2}+$ $\sin ^{2} \phi\left|\left\langle\psi|y| \psi^{\prime}\right\rangle\right|^{2}$. Averaging over all the possible $\phi$ form 0 to $2 \pi$, we obtain $\left(\left|\left\langle\psi|x| \psi^{\prime}\right\rangle\right|^{2}+\left|\left\langle\psi|y| \psi^{\prime}\right\rangle\right|^{2}\right) / 2$. For in-plane illumination, the angle $\phi$ is fixed (normal to the propagation of incident light in the plane $x, y$ ), and averaging Eq. (12) over $\theta$ in the interval $(-\pi / 2, \pi / 2)$, we obtain $\cos ^{2} \phi\left|\left\langle\psi|x| \psi^{\prime}\right\rangle\right|^{2} / 2+$ $\sin ^{2} \phi \mid\left\langle\left.\left\langle|y| \psi^{\prime}\right\rangle\right|^{2} / 2+\right|\left\langle\left.\left\langle|z| \psi^{\prime}\right\rangle\right|^{2} / 2\right.$ with only one term being nonzero. For instance, if the quantum number that changes is $n_{y}$, then the squared matrix element is $\sin ^{2} \phi \mid\left\langle\left.\left\langle|y| \psi^{\prime}\right\rangle\right|^{2} / 2\right.$ with lobes in the $\phi= \pm \pi / 2$ that correspond to light beams directed along the $x$-axis. In the case of in-plane illumination, it may be that the polarization is fixed, usually either $\theta=0$ or $\theta=\pi / 2$, and that the beam direction is also fixed. In this case, Eq. (12) must be used without any averaging.

\subsection{Calculation of the absorption coefficients}

As mentioned previously, the only permitted transitions involve the change of a single quantum number. This quantum number may correspond to a final state associated to either a bound or extended one-dimensional wavefunction. The other two quantum numbers remain unchanged and may correspond to bound or extended one-dimensional wavefunctions.

Photon absorption implies that the initial state is filled with one electron. As there are many QDs, a filling factor $f_{n x, n y, n z}$ can be defined for each state. This is usually a Fermi factor associated with a Fermi level $E_{F ; n x, n y, n z}$. In most cases, only the fundamental state $(1,1,1)$ is filled with electrons to a substantial extent. These electrons often come from donors introduced by doping. If the doping is too strong or under some other conditions, the first excited state may become partly filled. All the other states, including those in the CB, are essentially empty. Usually, the fundamental state where $E_{\text {line }}$ is, in each instance, the energy difference between the final and initial state in question. The number of totally BS in the QD is finite and usually restricted to few states. The factor 2 accounts for spin degeneracy. Note that $f_{1,2,1}=f_{2,1,1}$ because of symmetry.

Because only a single quantum number is allowed to change in each transition, we have $\alpha_{1,1,1}^{n x, n y, n z}=\alpha_{1,1,1}^{n x, 1,1}$ or $\alpha_{1,1,1}^{i n x, n y, n z}=\alpha_{1,1,1}^{1, n y, 1}$ or $\alpha_{1,1,1}^{\prime n x, n y, n z}=\alpha_{1,1,1}^{1,1, n z}$, the first two factors being identical because of the equality $a=b$. Hereafter, the notation $\alpha_{u ; 1}^{\prime n}$ will be used for transitions from the fundamental state to a final state in which the quantum number in the $u$ coordinate ( $u=x, y$, or $z$ ) has changed to $n^{\prime}$. It should be mentioned that $\alpha_{u ; n}^{n^{\prime}}$ is affected by the polarization factors studied in Section 3.2. The matrix elements appearing in the $\alpha_{u ; n}^{\prime \prime \prime}$ can be found in Eq. (A3) in the Appendix.

We now consider transitions from the fundamental or the first excited state to ESs. Because the initial states are bound in three dimensions and only one quantum number may vary per transition event, absorption only occurs involving final states that are extended in only one dimension. Let us assume that it is the $x$ dimension and calculate, using Eq. (6), the density $N\left(E_{x}\right)$ of ESs per unit of energy.

$$
N\left(E_{x}\right)=\frac{1}{\Delta k_{\text {even }}} \frac{d k_{x}}{d E_{x}}=\frac{1}{\Delta k_{\text {odd }}} \frac{d k_{x}}{d E_{x}}=\frac{L m_{\mathrm{c}}^{1 / 2}}{2^{1 / 2} \hbar \pi} E_{x}^{-1 / 2}
$$

The energy of the absorbed photons $E_{\text {line }}$ is now

$$
E_{\text {line }}=E_{k x, 1,1}-E_{1,1,1}=E_{x}-E_{x ; 1}
$$

where $E_{x, 1}$ represents the energy corresponding to the one-dimensional fundamental state. The same can be said for the first excited state, mutatis mutandis.

For the transition from a BS to a state extended in one dimension, we use the notation $\alpha_{u ; n}^{\prime}(k)$, where $n$ is the initial value of the changing quantum number with corresponding coordinate $u$. 
The absorption coefficient for transitions from a BS to all ESs is then

$$
\begin{aligned}
& \alpha_{u, n}^{E S}(k)=2 f_{n} \sum_{k}\left(1-f_{C}\right) E \alpha_{u ; n}^{\prime}(k) \delta\left(E_{\text {line }}-E\right) \\
& \cong 2 f_{n} \int N\left(E_{u}\right) E \alpha_{n ; u}^{\prime}(k) \delta\left(E_{\text {line }}-E\right) \mathrm{d} E \\
& \quad=2 f_{n} N\left(E_{\text {line }}+E_{u ; n}\right) E_{\text {line }} \alpha_{u ; n}^{\prime}\left(k_{u}\left(E_{\text {line }}+E_{u, n}\right)\right)
\end{aligned}
$$

Taking into account that only the fundamental and first excited states have appreciable probability of occupation; we can write

$$
\alpha^{E S}=\sum_{u=x, y} \sum_{n=1,2} 2 f_{n u, 1,1} \sum_{k_{u}} N\left(E_{\text {line }}+E_{u ; n}\right) E_{\text {line }} \alpha_{u ; n}^{\prime}\left(k_{u l}\left(E_{\text {line }}+E_{u, n}\right)\right)
$$

where the sums must take into account all the combinations of possible transitions.

The matrix elements necessary for the calculation of the $\alpha_{u ; n}^{\prime}(k)$ are presented in Eqs. (A4) and (A5) in the Appendix.

\section{DISCUSSION}

Let us apply the preceding calculations to IB material of the IB cell described as SB in [18] and as S3 in [19]. This IB cell has achieved, at low temperatures, an open circuit voltage very close to the GaAs bandgap [19]. The parameters characterizing these QDs are listed in Table I. The data sources are listed in the third column. Also included are the potential well depth and effective masses calculated in [13] and [16], respectively. These parameters

Table I. Parameters defining the quantum dots.

\begin{tabular}{lll}
\hline $\mathrm{a}(\mathrm{m})$ & $8.00 \mathrm{E}-09$ & TEM \\
$\mathrm{c}(\mathrm{m})$ & $3.00 \mathrm{E}-09$ & TEM \\
$m_{\mathrm{cb}}$ InAs & 0.0294 & {$[16]$} \\
$m_{\mathrm{cb}}$ GaAs & 0.0613 & {$[16]$} \\
$U_{\mathrm{cb}}(\mathrm{eV})$ & 0.473 & {$[13]$} \\
$n_{\mathrm{ref}}$ & 3.5 & {$[17]$} \\
$F$ & 0.1024 & AFM \\
$N_{1}\left(\mathrm{~cm}^{-1}\right)$ & 125000 & Design \\
\hline
\end{tabular}

TEM, transmission electron microscopy; AFM, atomic force microscopy. take into account the important QD strain effects and have been shown to be in agreement with absorption peaks observed in quantum efficiency (QE) measurements[7].

For these parameters, previous calculations have revealed four bound $X(x)$ and $Y(y)$ functions and two bound $Z(z)$ functions (see the specific procedure in [8]). The energy levels corresponding to these BSs relative to the square potential formed by the CB bottom are show in Figure 1. ESs are not shown. In the figure, the energy origin is at the GaAs CB bottom. The fundamental state is the $(1,1,1)$ state with an energy of $0.20994 \mathrm{eV}$ above the well $\mathrm{CB}$ bottom $(0.26306 \mathrm{eV}$ below the GaAs CB

bottom). The first excited state is $(1,2,1)$ or $(2,1,1)$ with energy of $0.31044 \mathrm{eV}$ above the well $\mathrm{CB}$ bottom ( 0.16256 below the GaAs CB bottom).

\subsection{Absorption by transitions between bound states}

In Table II, we present the absorption lines between BS, the matrix elements obtained by application of Eq. (A3) and the $\alpha^{\prime}$ s obtained by application of Eq. (10). In this table, the spin degeneracy is not taken into account in calculating $\alpha_{u ; n}^{\prime n}$, and the polarization factor is taken to be 1 .

Transitions between quantum numbers of the same parity are omitted ( $\alpha^{\prime}=0$ for these transitions, as discussed). $E_{\text {line }}$ depends only on the quantum number being changed. For instance, the transition $(1,1,1) \rightarrow(1,2,1)$ and the transition $(2,1,1) \rightarrow(2,2,1)$ have the same $E_{\text {line }}$.

Table III shows all possible transitions between BS under the assumption that only the fundamental level $(1,1,1)$ and the first excited level with states $(1,2,1)$ and $(2,1,1)$ are appreciably filled with electrons.

We consider four examples of illumination: an unpolarized beam in the $z$ direction (denoted $z$ ), an unpolarized beam in the $x$ direction (x), a $y$-polarized beam in the $x$ direction $(x / y)$, and a $z$-polarized beam in the $x$ direction $(x / z)$. Using the formulas in Section 3.2, we present the polarization coefficient of the transition. The

\begin{tabular}{|c|c|c|c|c|c|}
\hline \multicolumn{5}{|c|}{$n_{x}, n_{y}$} & \multirow{2}{*}{$\frac{n_{z}}{1 \rightarrow 2}$} \\
\hline Transition & $1 \rightarrow 2$ & $2 \rightarrow 3$ & $3 \rightarrow 4$ & $1 \rightarrow 4$ & \\
\hline$E_{\text {line }}(\epsilon V)$ & 0.101 & 0.159 & 0.175 & 0.434 & 0.312 \\
\hline$\left|\left\langle\phi|y| \phi^{\prime}\right\rangle\right|^{2} / 4 a b$ & 0.04889 & 0.06154 & 0.05736 & $1.9451 \times 10^{-4}$ & 0.01281 \\
\hline$\alpha_{u ; n}^{i n}$ & 5.17734 & 10.2849 & 10.5887 & 0.0890091 & 4.20973 \\
\hline
\end{tabular}
values of $\alpha^{B S}$ are calculated according to Eq. (13) using the values in Table II. The states in the sum are

Table II. Bound-to-bound level transitions. 
Table III. Bound-to-bound state transitions and their polarization factors for several polarization modalities.

\begin{tabular}{cccccc}
\hline Start & End & $z$ & $x$ & $x / y$ & $x / z$ \\
\hline $111 \rightarrow$ & 121 & $1 / 2$ & $1 / 2$ & 1 & 0 \\
& 211 & $1 / 2$ & 0 & 0 & 0 \\
& 141 & $1 / 2$ & $1 / 2$ & 1 & 0 \\
& 411 & $1 / 2$ & 0 & 0 & 0 \\
& 112 & 0 & $1 / 2$ & 0 & 1 \\
$121 \rightarrow$ & 131 & $1 / 2$ & $1 / 2$ & 1 & 0 \\
& 221 & $1 / 2$ & 0 & 0 & 0 \\
& 421 & $1 / 2$ & 0 & 0 & 0 \\
& 122 & 0 & $1 / 2$ & 0 & 1 \\
$211 \rightarrow$ & 311 & $1 / 2$ & 0 & 0 & 0 \\
& 221 & $1 / 2$ & $1 / 2$ & 1 & 0 \\
& 241 & $1 / 2$ & $1 / 2$ & 1 & 0 \\
& 212 & 0 & $1 / 2$ & 0 & 1 \\
\hline
\end{tabular}

determined with the help of Table III. Each $\alpha_{u ; n}^{\prime n^{\prime}}$ in the sum is multiplied by the polarization factor corresponding to the type of illumination also found in Table III. Several terms in the sum may correspond to the same line. The absorption spectrum is a train of Dirac deltas. The Dirac delta distribution is usually approximated by a more distributed function to account for the statistical contribution of QDs of variable dimensions in the sample. We use a Gaussian distribution,

$$
\delta\left(E-E_{\text {line }}\right) \cong \frac{1}{\sigma \sqrt{\pi}} \exp \left(-\frac{\left(E-E_{\text {line }}\right)^{2}}{\sigma^{2}}\right)
$$

where $\sigma$ represents the energy dispersion. This dispersion depends on the regularity of the sample, although the integrated values are independent of $\sigma$. The energy dispersion has been set to $0.025 \mathrm{eV}$.

Optimal filling for IB solar cells requires the fundamental state to be partially filled and the first excited state to be empty [20]. For QDIPs, the fundamental state should be totally filled, and the first excited state should be empty. Focusing our study on IB solar cells, we present in Figure 2 the absorption coefficients for the case (A) $f_{1,1,1}=0.5, f_{1,2,1}=f_{2,1,1}=0$ corresponding to optimal doping of the IB region and for the case (B) $f_{1,1,1}=1, f_{1,2,1}=f_{2,1,1}=0.5$ corresponding to overdoping. The illumination conditions are specified in the figure caption.

Compared with the first case, the second case leads to a much stronger absorption and exhibits a line at $0.159 \mathrm{eV}$ absent in the first case. What is more, there is a reduction in the intensity of the first line relative to the last line. The different beam directions and polarizations have a strong influence on the absorption spectrum. In particular, the in-plane unpolarized illumination reduces the absorption by half at the $0.101 \mathrm{eV}$ line with
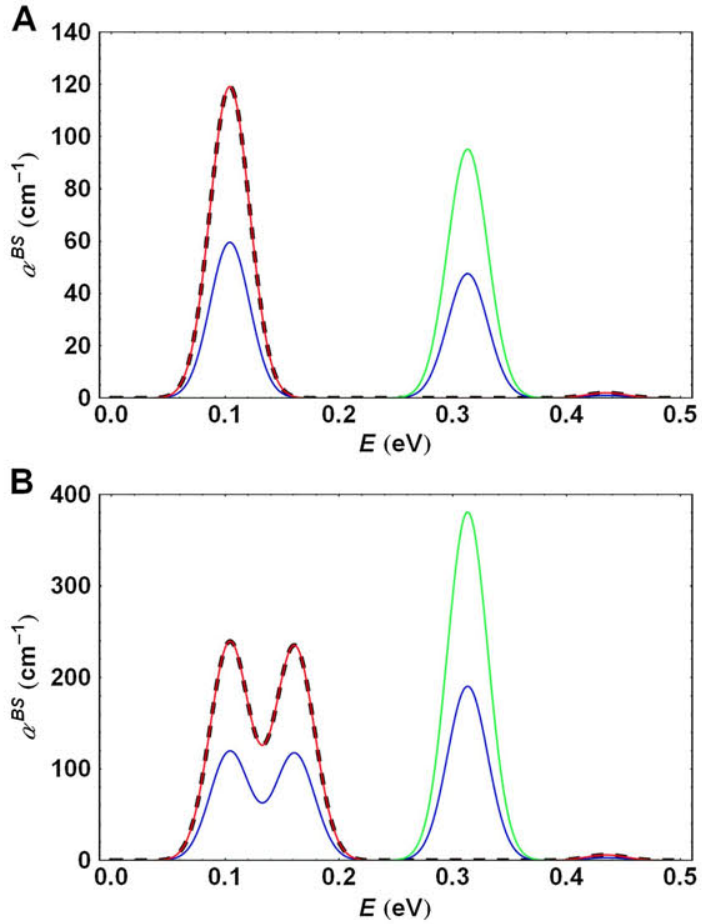

Figure 2. Absorption coefficient due to transitions between bound states. Dashed black curve, $z$ (normal) unpolarized beam; blue curve, $x$ unpolarized beam; red curve, $y$-polarized $x$-beam; green curve, $z$-polarized $x$-beam. (A) the fundamental $(1,1,1)$ state is filled to $50 \%$. (B) the fundamental state is filled to $100 \%$, and the first excited states $(1,2,1)$ and $(2,1,1)$ are filled to $50 \%, \sigma=0.025 \mathrm{eV}$.

respect to the normal unpolarized illumination but produces an additional absorption at the $0.312 \mathrm{eV}$ line.

\subsection{Absorption by transitions between bound and extended states}

We present in Table IV the possible absorption transitions if the only initial states with electrons are again the

Table IV. Bound-to-extended state transitions for several polarization modalities and their polarization factors.

\begin{tabular}{lccccc}
\hline Beam/pol. & & $z$ & $x$ & $x / y$ & $x / z$ \\
\hline $111 \rightarrow$ & $1 \mathrm{k} 1$ & $1 / 2$ & $1 / 2$ & 1 & 0 \\
& $\mathrm{k} 11$ & $1 / 2$ & 0 & 0 & 0 \\
\multirow{3}{*}{$121 \rightarrow$} & $11 \mathrm{k}$ & 0 & $1 / 2$ & 0 & 1 \\
& $1 \mathrm{k} 1$ & $1 / 2$ & $1 / 2$ & 1 & 0 \\
& $\mathrm{k} 21$ & $1 / 2$ & 0 & 0 & 0 \\
$211 \rightarrow$ & $12 \mathrm{k}$ & 0 & $1 / 2$ & 0 & 1 \\
& $2 \mathrm{k} 1$ & $1 / 2$ & $1 / 2$ & 1 & 0 \\
& $\mathrm{k} 11$ & $1 / 2$ & 0 & 0 & 0 \\
& $21 \mathrm{k}$ & 0 & $1 / 2$ & 0 & 1 \\
\hline
\end{tabular}


fundamental and the first excited states. The absorption coefficient is given by Eq. (17) summing over all the transitions found in this table. The matrix elements are given by Eq. (A4) when the starting changing quantum number is 1 and by Eq. (A5) when the starting changing quantum number is 2 . The $\alpha_{u ; n}^{\prime}$ is calculated with Eq. (10). The polarization factors are also in Table IV. The functions $\alpha_{u, n}^{E S}(k)$ defined in Eq. (16) are zero for photon energies below $E_{u ; n}$ but are nonzero at this energy, that is, they show a discontinuity at their onset. To account for this, we can multiply them by a step function starting at $E_{u ; n}$. However, in the same way, we have used a Gaussian function, instead of a Dirac delta, to account for the dispersion of shapes of the QDs; here, we can replace the step function with

$$
H\left(E-E_{\text {line }}\right) \cong\left[\exp \left(-\frac{E-E_{u ; n}}{2 \sigma}\right)+1\right]^{-1}
$$

with $\sigma=0.025 \mathrm{eV}$. In Figure 3, we present the absorption coefficients $\alpha^{B S}+\alpha^{E S}$ for the cases (A) $f_{1,1,1}=0.5$, $f_{1,2,1}=f_{2,1,1}=0$ and (B) $f_{1,1,1}=1, f_{1,2,1}=f_{2,1,1}=0.5$.

To the left of each graph, we observe the BS $\rightarrow$ BS absorption lines $\alpha^{B S}$ already drawn in Figure 2. To the right, the $\mathrm{BS} \rightarrow \mathrm{ES}$ continuous absorption spectra $\alpha^{E S}$ is
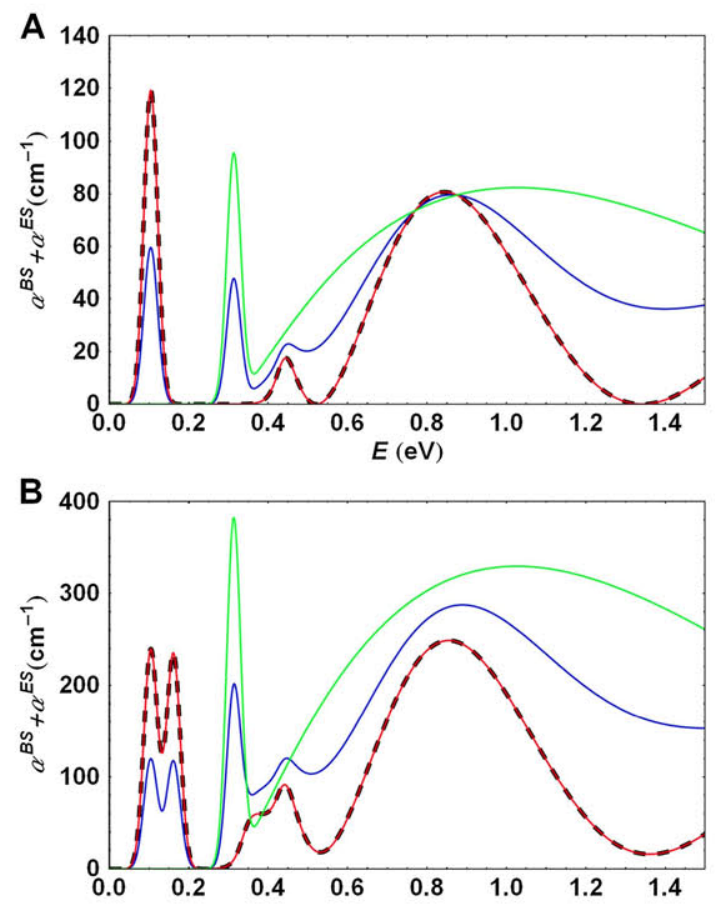

Figure 3. Absorption coefficient due to transitions between bound-to-bound and bound-to-extended states. Dashed black curve, $z$ (normal) unpolarized beam; blue curve, $x$ unpolarized beam; red curve, $y$-polarized $x$-beam; green curve, $z$-polarized $x$-beam. (A) the fundamental $(1,1,1)$ state is filled to $50 \%$.

(B) the fundamental state is filled to $100 \%$, and the first excited states $(1,2,1)$ and $(2,1,1)$ are filled to $50 \% . \sigma=0.025 \mathrm{eV}$. shown. Indeed, it continues beyond $1.5 \mathrm{eV}$. However, above this energy, the radiation is filtered by $\mathrm{VB} \rightarrow \mathrm{CB}$ transitions either at the emitter or within the IB region. Thus, the $\mathrm{BS} \rightarrow \mathrm{ES}$ absorption beyond $1.5 \mathrm{eV}$ becomes negligible.

In Figure 3, we observe that the absorption is higher for the case with more electrons, as expected. This does not imply that the case with more electrons would be optimal for an IB solar cell because the fundamental state must be only partially filled to be able to accept electrons generated from the VB [20]. The intraband spectrum covers almost the entire spectrum with the exception of some very low photon energies. Actually, the absorption we have calculated is a variation of free electron absorption acting from the BSs, which are detached from the CB ESs. As in the case of free electrons, this absorption is not directly associated with electron-hole generation and therefore is not appreciated as a current, except in some special devices such as IB solar cells and QDIPs when the appropriate conditions are met.

The magnitude of this absorption is rather small when compared with the $\mathrm{VB} \rightarrow \mathrm{CB}$ absorption (which is over three orders of magnitude stronger). However, it is comparable with that observed for the intraband transitions leading to the sub-bandgap internal QE calculated in [7]. The detailed balance counterpart of the absorption calculated here is the photon emission. Knowing this, we can undertake realistic detailed balance calculations. This paper is intended to be a necessary step towards their formulation.

Going one step further toward a detailed balance study, we give in Table $\mathrm{V}$ the gross generation calculated with Eq. (8) for the extraterrestrial (air mass zero, AM0) spectrum of solar photons, that is (see e.g. in [21]),

$$
\frac{\mathrm{d} N_{\mathrm{ph}}}{\mathrm{d} E}=\frac{1}{46000} \frac{2 \pi}{c^{2} h^{3}} \frac{E^{2}}{\exp \left(\frac{E}{k_{B} 5760}\right)-1}
$$

The value 5760 is the sun's photosphere temperature (in $\mathrm{K}$ ), and the term $1 / 4600$ is the photon dilution at the sun-earth distance. The term gross generation is used because recombination, that is, transitions with the emission of a photon, is not accounted for.

Table V. Generation in $\mathrm{A} \mathrm{cm}^{-3}$ due to air mass zero illumination for transitions form a full $(1,1,1)$ state to the transition-connected states.

\begin{tabular}{ccccc}
\hline & $v$ & $x$ & $x y$ & $x z$ \\
\hline 121 & 0.0081 & 0.0081 & 0.0162 & 0.0000 \\
211 & 0.0081 & 0.0000 & 0.0000 & 0.0000 \\
141 & 0.0018 & 0.0018 & 0.0036 & 0.0000 \\
411 & 0.0018 & 0.0000 & 0.0000 & 0.0000 \\
112 & 0.0000 & 0.0503 & 0.0000 & 0.1006 \\
$1 \mathrm{k} 1$ & 1.8274 & 1.8274 & 3.6548 & 0.0000 \\
$\mathrm{k} 11$ & 1.8274 & 0.0000 & 0.0000 & 0.0000 \\
$11 \mathrm{k}$ & 0.0000 & 3.8077 & 0.0000 & 7.6155 \\
\hline
\end{tabular}


In Table $\mathrm{V}$, the generation rate is multiplied by the electron charge so that the calculated values are in $\mathrm{A}$ $\mathrm{cm}^{-3}$. Multiplying by the volume of the device gives the current generation. The values in the table correspond to the $(1,1,1)$ state being totally filled of electrons and all other states being empty. For partial filling, we must multiply the values in this table by the filling factor of the fundamental level. For transitions to the continuous spectrum, the transitions stop at $1.5 \mathrm{eV}$. The radiation is considered to be filtered for higher energies as discussed previously. It can be seen that the transitions to the continuous spectrum are significantly stronger than those to the BSs.

\section{CONCLUSIONS}

This paper uses and further develops the separation of variables model for QDs in the frame of $\mathrm{k} \cdot \mathrm{p}$ theory already presented elsewhere [8]. The model is used to calculate the absorption coefficient for transitions between BSs and ESs. Analytical expressions of the optical matrix elements corresponding to these transitions are given in this paper (Appendix). Because of the small overlap between the ES and BS wavefunctions, the matrix elements are small. However, because of the numerous states of the continuous ES spectrum, the aggregate contribution of all transitions leads to a finite absorption coefficient that is very important. The BS ES transitions have been introduced into the frame of our separation of variables $\mathrm{k} \cdot \mathrm{p}$ model for the first time. For completeness, we also present the BS $\rightarrow$ BS transitions that were already introduced in a previous letter [8] but here with the analytical expressions included explicitly in the Appendix.

In our separation of variables model, wavefunctions may be extended in zero, one, two, or three dimensions and bound in the rest. Our model establishes that the only possible transitions are those that link states differing in only one (out of three) quantum number. Therefore, the continuous spectrum states that can receive an electron by photon absorption from a BS electron are extended in a single dimension.

When comparing the VB $\rightarrow \mathrm{BS}$ interband absorption [7] and the $\mathrm{BS} \rightarrow \mathrm{CB}$ intraband absorption presented here, we find that they are of similar strength. It is concluded that such transitions are strong enough as to provide a quick path to the $\mathrm{CB}$ for electrons pumped from the valence to the IB. In the previous work [7], this result had been assumed implicitly in the calculation of the $\mathrm{QE}$, the accuracy of the assumption being inferred from the good agreement between the calculated and measured QEs.

The absorption may have been expected to be stronger than for the interband transitions calculated in [7] because the valence band states have a zero component in the CB, and therefore, the transitions must occur with the $8 \%$ projection of the $\mathrm{CB}$ wavefunctions already mentioned. However, this is compensated by the relatively large number of heavy hole BSs in the VB.

\section{REFERENCES}

1. Luque A, Martí A. Increasing the efficiency of ideal solar cells by photon induced transitions at intermediate levels. Physical Review Letters 1997; 78(26): 5014-5017.

2. Martí A, Cuadra L, Luque A. Quantum dot intermediate band solar cell. IEEE: Proc. 28th IEEE Photovoltaics Specialists Conference, New York, 2000.

3. Luque A, Martí A, Stanley C, López N, Cuadra L, Zhou D, Mc-Kee A. General equivalent circuit for intermediate band devices: potentials, currents and electroluminescence. Journal of Applied Physics 2004; 96(1): 903-909.

4. Berryman KW, Lyon SA, Segev M. Mid-infrared photoconductivity in InAs quantum dots. Applied Physics Letters 1997; 70(14): 1861-1863.

5. Liu HC, Duboz JY, Dudek R, Wasilewski ZR, Fafard S, Finnie P. Quantum dot infrared photodetectors. Physica E: Low-dimensional Systems and Nanostructures 2003; 17: 631-633.

6. Datta S. Quantum Phenomena. Addison Wesley: Reading (Mass), 1989.

7. Luque A, Martí A, Antolín E, Linares PG, Tobías I, Ramiro I, Hernandez E. New Hamiltonian for a better understanding of the quantum dot intermediate band solar cells. Solar Energy Materials and Solar Cells 2011; 95(8): 2095-2101.

8. Luque A, Marti A, Antolin E, Garcia-Linares $P$. Intraband absorption for normal illumination in quantum dot intermediate band solar cells. Solar Energy Materials and Solar Cells 2010; 94: 2032-2035.

9. Sugawara M. Self-assembled InGaAs/GaAs quantum dots. Academic Press: San Diego, 1999.

10. Lazarenkova OL, Balandin AA. Miniband formation in a quantum dot crystal. Journal of Applied Physics 2001; 89(10): 5509-5515.

11. Shao Q, Balandin AA, Fedoseyev AI, Turowski M. Intermediate-band solar cells based on quantum dot supracrystals. Applied Physics Letters 2007; 91(16): 163503.

12. Popescu V, Bester G, Hanna MC, Norman AG, Zunger A. Theoretical and experimental examination of the intermediate-band concept for strain-balanced (In,Ga)As/Ga(As,P) quantum dot solar cells. Physical Review B 2008; 78: 205321.

13. Popescu V, Bester G, Zunger A. Coexistence and coupling of zero-dimensional, two-dimensional, and continuum resonances in nanostructures. Physical Review B 2009; 80(4): 045327.

14. Bastard G, Ziemelis UO, Delalande C, Voos M, Gossard AC, Wiegmann W. Bound and Virtual Bound-States in Semiconductor Quantum Wells. Solid State Communications 1984; 49(7): 671-674. 
15. Popescu V, Bester G, Zunger A. Strain-induced localized states within the matrix continuum of selfassembled quantum dots. Applied Physics Letters 2009; 95(2): 023108.

16. Linares PG, Marti A, Antolin E, Luque A. III-V Compound semiconductor screening for implementing quantum dot intermediate band solar cells. Joumal of Applied Physics 2011; 109: 014313.

17. Sze SM. Physics of Semiconductor Devices. John Wiley \& Sons: New York, 1981.

18. Antolín E, Marti A, Farmer CD, Linares PG, Hernández E, Sánchez AM, Ben T, Molina SI, Stanley CR, Luque A. Reducing carrier escape in the InAs/GaAs quantum dot intermediate band solar cell. Journal of Applied Physics 2010; 108(6): 064513.

19. Antolín E, Martí A, Linares PG, Ramiro I, Hernández E, Farmer CD, Ben T, Luque A. Advances in Quantum Dot Intermediate Band Solar Cells. IEEE: Proc.25 Photovoltaic Specialists Conference, Honolulu, 2010.

20. Luque A, Marti A. On the partial filling of the intermediate band in ib solar cells. IEEE Transactions on Electron Devices Jun 2010; 57: 1201-1207.

21. Vos Ad. Endoreversible Thermodynamics of Solar Energy Conversion. Oxford University Press: New York, 1992.

\section{APPENDIX}

In this appendix, we define $\zeta^{2}=\left(2 m_{\mathrm{c}} / \hbar^{2}\right) U_{0}$. We use $k_{0}$ and $k_{1}$ corresponding to even and odd functions, respectively. These are either bound or extended; in the former case, they take any of the permitted discrete values. The formulae that follow are the result of extensive but straightforward calculations. The calculations are made for the $x$-coordinate but are equally valid for the other coordinates, mutatis mutandis.

\section{A1 Norm of bound functions}

Denoting the width of the potential well for the dimension in consideration as $d$, the one- dimensional norms $N_{B 0}$ and $N_{B 1}$ for respective even and odd functions are is given by

$$
\begin{aligned}
& N_{B 0}^{2}=2 \int_{0}^{\infty} \alpha(x)^{2} \mathrm{~d} x=\mathrm{d}+\frac{\mathrm{d} \cos (k \mathrm{~d}) \sin (k \mathrm{~d})}{k \mathrm{~d}}+\frac{\mathrm{d} \cos ^{2}(k \mathrm{~d})}{\sqrt{\zeta^{2} \mathrm{~d}^{2}-k^{2} \mathrm{~d}^{2}}} \\
& N_{B 1}^{2}=2 \int_{0}^{\infty} \alpha(x)^{2} \mathrm{~d} x=\mathrm{d}-\frac{\mathrm{d} \cos (k \mathrm{~d}) \sin (k \mathrm{~d})}{k \mathrm{~d}}+\frac{\mathrm{d} \sin ^{2}(k \mathrm{~d})}{\sqrt{\zeta^{2} \mathrm{~d}^{2}-k^{2} \mathrm{~d}^{2}}}
\end{aligned}
$$

The expression $\alpha(x)$ contains a cosine or a sine function flanked by fading exponential functions with coefficients properly matched to assure continuity. The continuity of the derivative is assured if $k$ fulfills Eq. (3).

The three-dimensional norm is the product of three onedimensional norms.

\section{A2 Norm of extended functions}

The periodic conditions assumed for the one-dimensional wavefunctions in a segment of length $2 L$ determine the continuous spectrum formed by the set of permitted values of $k=j \pi / 2 L$, where $j$ is an integer. For the proper choice of $\theta$ in Eq. (4), the continuity of the functions (and their derivatives) at $x=d$ is assured, but this choice will not affect the result. For the unbounded even ( $j$ odd) and odd ( $j$ even) functions, the norms are respectively

$$
\begin{aligned}
\mathrm{N}_{\mathrm{Ext} 0}^{2} & =2\left[\int_{0}^{d} \cos ^{2}(k x) d x+\int_{d}^{L} \cos ^{2}\left(\frac{j}{2} \frac{x-\theta}{L-\theta}\right) d x\right] \\
= & d+\frac{d \cos (k d) \sin (k d)}{k d}+(L-d)-\frac{(L-\theta)}{j \pi} \sin \left(j \pi \frac{d-\theta}{L-\theta}\right) \\
N_{\mathrm{Ex} 11}^{2} & =2\left[\int_{0}^{d} \sin ^{2}(k x) d x+\int_{d}^{L} \sin ^{2}\left(\frac{j \pi x}{2} \frac{x-\theta}{L-\theta}\right) d x\right] \\
= & d-\frac{d \cos (k d) \sin (k d)}{k d}+(L-d)+\frac{(L-\theta)}{j \pi} \sin \left(j \pi \frac{d-\theta}{L-\theta}\right)
\end{aligned}
$$

For very large $L$ (as compared with $d, 1 / k$, and $\theta$ ), $N^{2}=L$ independent of the $j$ taken (provided $j \pi<<L$ ). Usually, $L=1 \mathrm{~cm}$ is more than sufficient.

\section{A3 Matrix elements for bound functions}

The matrix elements must link one even and one odd function. The matrix element is

The subindex 0 corresponds to the even solution, the subindex 1 to the odd solution.

\section{A4 Matrix elements for extended functions}

We are actually considering the matrix elements linking bound and extended functions. We can consider two cases: the bound function is even and the extended function is odd and vice versa. Starting with the first case,

For the case of transitions of an odd BS to an even ES, the formula is 


$$
\begin{aligned}
\left\langle\alpha_{0}|x| \alpha_{1}\right\rangle= & 2 d^{2} \frac{k_{0} d \sin \left(k_{0} d\right)\left[\left(k_{0}^{2} d^{2}-k_{1}^{2} d^{2}\right) \sin \left(k_{1} d\right)-2 k_{1} d \cos \left(k_{1} d\right)\right]}{\left(k_{0}^{2} d^{2}-k_{1}^{2} d^{2}\right)^{2} N_{B 0} N_{B 1}} \\
& +2 d^{2} \frac{\cos \left(k_{0} d\right)\left[\left(k_{0}^{2} d^{2}+k_{1}^{2} d^{2}\right) d^{2} \sin \left(k_{1} d\right)+k_{1}\left(k_{0}^{2}-k_{1}^{2}\right) d^{3} \cos \left(k_{1} d\right)\right]}{\left(k_{0}^{2} d^{2}-k_{1}^{2} d^{2}\right)^{2} N_{B 0} N_{B 1}} \\
& +2 d^{2} \frac{\left(1+\sqrt{\zeta^{2} d^{2}-k_{0}^{2} d^{2}}+\sqrt{\left.\zeta^{2} d^{2}-k_{1}^{2} d^{2}\right) \cos \left(k_{0} d\right) \sin \left(k_{1} d\right)}\right.}{\left(\sqrt{\zeta^{2} d^{2}-k_{0}^{2} d^{2}}+\sqrt{\zeta^{2} d^{2}-k_{1}^{2} d^{2}}\right)^{2} N_{B 0} N_{B 1}}
\end{aligned}
$$

$$
\begin{aligned}
\left\langle\alpha_{0}^{B S}|x| \alpha_{1}^{E S}\right\rangle= & 2 d^{2} \frac{k_{0} d \sin \left(k_{0} d\right)\left[\left(k_{0}^{2} d^{2}-k_{1}^{2} d^{2}\right) \sin \left(k_{1} d\right)-2 k_{1} d \cos \left(k_{1} d\right)\right]}{\left(k_{0}^{2} d^{2}-k_{1}^{2} d^{2}\right)^{2} N_{B 0} L^{1 / 2}} \\
& +2 d^{2} \frac{\cos \left(k_{0} d\right)\left[\left(k_{0}^{2} d^{2}+k_{1}^{2} d^{2}\right) d^{2} \sin \left(k_{1} d\right)+k_{1}\left(k_{0}^{2}-k_{1}^{2}\right) d^{3} \cos \left(k_{1} d\right)\right]}{\left(k_{0}^{2} d^{2}-k_{1}^{2} d^{2}\right)^{2} N_{B 0} L^{1 / 2}} \\
& +2 d^{2} \frac{\cos \left(k_{0} d\right) \sin \left(k_{1} d\right)\left[k_{0}^{2} d^{2}\left(1+\sqrt{\zeta^{2} d^{2}-k_{0}^{2} d^{2}}\right)-k_{1}^{2} d^{2}\left(-1+\sqrt{\zeta^{2} d^{2}-k_{0}^{2} d^{2}}\right)\right]}{\left(k_{0}^{2} d^{2}-k_{1}^{2} d^{2}\right)^{2} N_{B 0} L^{1 / 2}} \\
& +2 d^{2} \frac{\cos \left(k_{0} d\right) \sin \left(k_{1} d\right)\left[k_{0} d \cot \left(k_{0} d\right)\left(k_{0}^{2} d^{2}-k_{1}^{2} d^{2}-2 \sqrt{\zeta^{2} d^{2}-k_{0}^{2} d^{2}}\right)-2 d^{2} \zeta^{2}\right]}{\left(k_{0}^{2} d^{2}-k_{1}^{2} d^{2}\right)^{2} N_{B 0} L^{1 / 2}}
\end{aligned}
$$

$$
\begin{aligned}
\left\langle\alpha_{1}^{B S}|x| \alpha_{0}^{E S}\right\rangle= & 2 d^{2} \frac{k_{0} d \sin \left(k_{0} d\right)\left[\left(k_{0}^{2} d^{2}-k_{1}^{2} d^{2}\right) \sin \left(k_{1} d\right)-2 k_{1} d \cos \left(k_{1} d\right)\right]}{\left(k_{0}^{2} d^{2}-k_{1}^{2} d^{2}\right)^{2} N_{B 0} L^{1 / 2}} \\
& +2 d^{2} \frac{\cos \left(k_{0} d\right)\left[\left(k_{0}^{2} d^{2}+k_{1}^{2} d^{2}\right) d^{2} \sin \left(k_{1} d\right)+k_{1}\left(k_{0}^{2}-k_{1}^{2}\right) d^{3} \cos \left(k_{1} d\right)\right]}{\left(k_{0}^{2} d^{2}-k_{1}^{2} d^{2}\right)^{2} N_{B 0} L^{1 / 2}} \\
& +2 d^{2} \frac{\cos \left(k_{0} d\right) \sin \left(k_{1} d\right)\left[k_{0}^{2} d^{2}\left(-1+\sqrt{\zeta^{2} d^{2}-k_{1}^{2} d^{2}}\right)-k_{1}^{2} d^{2}\left(1+\sqrt{\zeta^{2} d^{2}-k_{1}^{2} d^{2}}\right)\right]}{\left(k_{0}^{2} d^{2}-k_{1}^{2} d^{2}\right)^{2} N_{B 0} L^{1 / 2}} \\
& +2 d^{2} \frac{\cos \left(k_{0} d\right) \sin \left(k_{1} d\right)\left[k_{0} d \cot \left(k_{0} d\right)\left(k_{1}^{2} d^{2}-k_{0}^{2} d^{2}-2 \sqrt{\zeta^{2} d^{2}-k_{1}^{2} d^{2}}\right)+2 d^{2} \zeta^{2}\right]}{\left(k_{0}^{2} d^{2}-k_{1}^{2} d^{2}\right)^{2} N_{B 0} L^{1 / 2}}
\end{aligned}
$$

